\title{
Alignment Considerations for Precise Large Area Imaging and EDS Mapping
}

Shangshang $\mathrm{Mu}^{1}$, Shawn Wallace ${ }^{2}$ and Jens Rafaelsen ${ }^{3}$

${ }^{1}$ EDAX Inc, A Division of Ametek, Materials Analysis Division, Draper, Utah, United States, ${ }^{2}$ EDAX Inc, A Division of Ametek, Materials Analysis Division, Mahwah, New Jersey, United States, ${ }^{3}$ EDAX, Fair Lawn, New Jersey, United States

SEM imaging and EDS mapping of geological and extraterrestrial materials usually require analyzing at relatively high magnifications to investigate microscale features and a representative area to minimize the heterogeneity induced by grain size and phase distributions across the entire sample (Figure 1). Modern SEM and EDS enable precise large area imaging and EDS mapping by collecting individual high magnification/resolution images and maps through a grid pattern over a large sample surface and stitching them into a montage [1]. The basic requirement for such applications is ensuring good alignment of edge features of individual images and maps with respect to neighbors. While it is possible to adjust focus with working distance (WD) or Z stage movement, it is also recommended to keep a constant working distance throughout the entire imaging/analyzing area to reduce errors. This study discusses relatively easy practices to check and satisfy alignment considerations in preparation for collecting large area images and EDS maps.

SEM image scan rotation and magnification reference are the major considerations for montage image alignment. The sample used for this alignment experiment was a piece of TEM square grids. A montage of SEM images that consists of multiple square grids was collected in a 2 x 2 stage field pattern before and after each fine tune for evaluating the alignment. By examining the squareness of the grids along the axes of the four quadrants which are the edges between individual images of the montage, the offsets can be identified and used as a guideline for scan rotation and magnification reference adjustments.

If the beam axis is not precisely aligned to the stage movement in the $\mathrm{X}$-axis, moving the stage along the $\mathrm{Y}$-axis will cause a position change in the $\mathrm{Y}$-axis as well. This can be easily visualized in the montage of square grids. Figure $2 \mathrm{a}$ and $2 \mathrm{~b}$ show the beam axis is rotated counterclockwise and clockwise relative to the stage motion direction, respectively, as indicated by the mismatch between the left half and the right half of the square grids along the vertical axis in red color. The scan rotation adjustment is necessary until the mismatch is eliminated (Figure 2c).

Once scan rotation is adjusted, the magnification reference width and height values need to be tuned in the EDS or scan generator software, so the stage moves exactly one field in $\mathrm{X}$ and $\mathrm{Y}$ directions. This procedure may be started from the direction that requires larger adjustment. The length of each side of the grids along the axes should be measured on screen to help identify and fix minor offsets. However, note that the SEM image width and stage movement may not be fully calibrated and that the offset can be magnification dependent. If the stage stops short or moves too far, the reference value is set too small or large, respectively. Figure $2 \mathrm{~d}$ shows that the stage movement is too far in the $\mathrm{Y}$ direction since the grids along the horizontal axis are heavily shrunk in the Y direction. As shown in Figure 2e, after decreasing the reference height value, the stage moves exactly one field in the Y direction. This figure also indicates that the stage moves a little far in the $\mathrm{X}$ direction as the horizontal side of the grids along the vertical axis is measured slightly shorter than the vertical side. This can be adjusted by marginally decreasing the reference width value (Figure 2f). For EDS mapping, the scale bar on the SEM is not necessarily calibrated along with the stage. This magnification reference adjustment on the EDS side compensates for the $\mathrm{x} / \mathrm{y}$ scaling. For large area imaging on the SEM side only, the scale bar calibration is still required. The scan 
rotation and magnification reference adjustments are interactive and may be repeated multiple times until an optimal montage alignment is achieved.

For the constant working distance consideration, the sample should be polished flat and mounted parallel to the stage motion in the first place. Furthermore, the $x-y$ motion of the stage should be perpendicular to the beam axis and the inclination angle between the stage normal and the beam axis needs to be evaluated. For this experiment, we chose three positions at the edge of the stage of a FEI Teneo SEM and recorded the stage coordinates $\mathrm{P} 0=(-31.5413,17.5088,60.4446), \mathrm{P} 1=(2.7049,-36.4853,60.4993)$, and $\mathrm{P} 2=$ $(32.7951,14.9375,60.3005)$ (in $\mathrm{mm})$. The $\mathrm{Z}$ of each position was determined by using $\mathrm{Z}$ stage motion only to focus a spherical dirt particle with a diameter of approximately $500 \mathrm{~nm}$ on the stage at $20000 \mathrm{X}$ magnification. A maximum $\mathrm{Z}$ variation of $0.2 \mathrm{~mm}$ was observed, representing a minor stage inclination of $0.2^{\circ}$ by calculation. Note that if a flat sample is not mounted parallel to the stage, a rotation and tilt can be used to achieve a surface perpendicular to the beam.
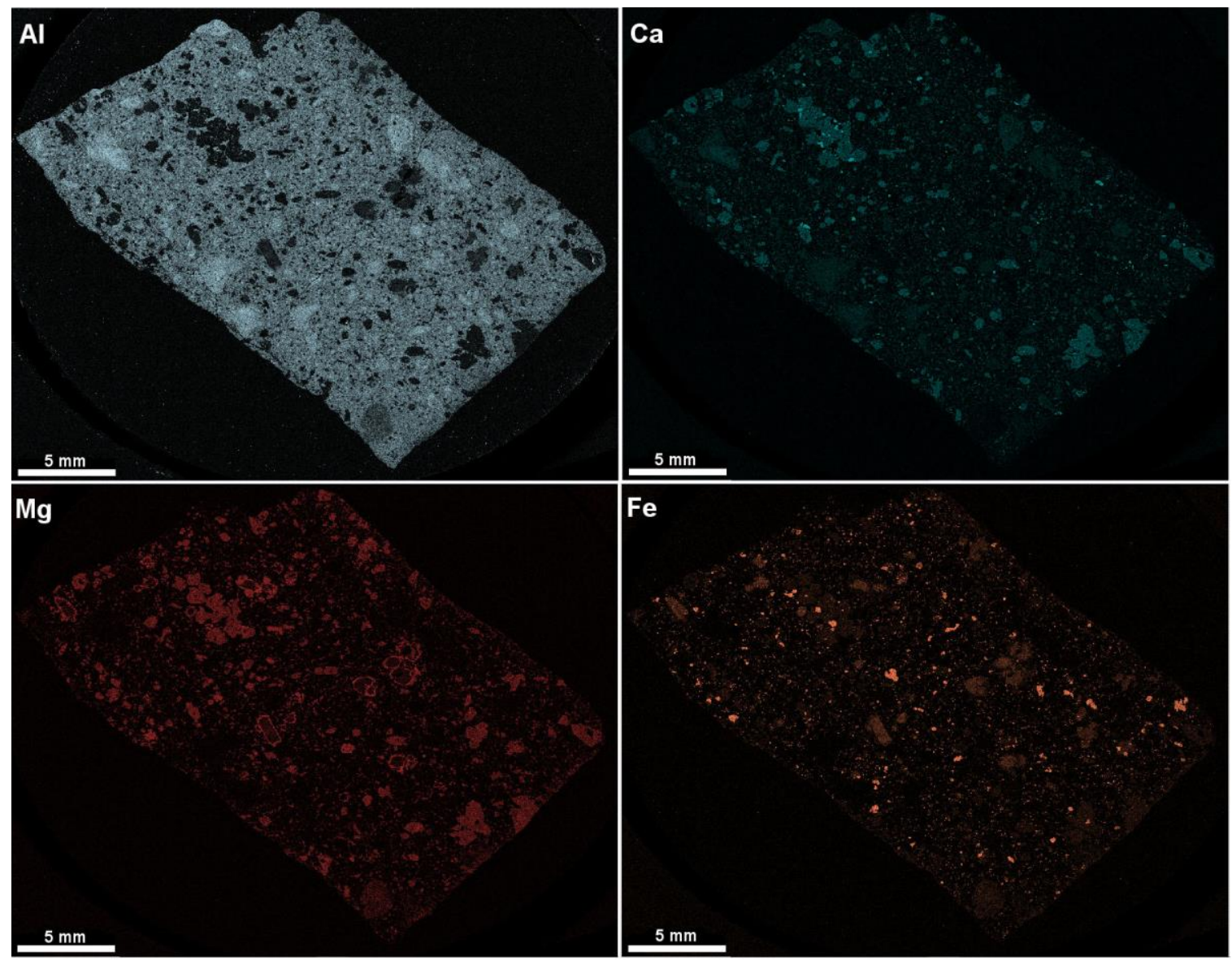

Figure 1. Montage EDS maps of an entire $2.5 \times 1.5 \mathrm{~cm}$ Hornblende Basalt section acquired using EDAX APEX 2.0 software show heterogeneity. 


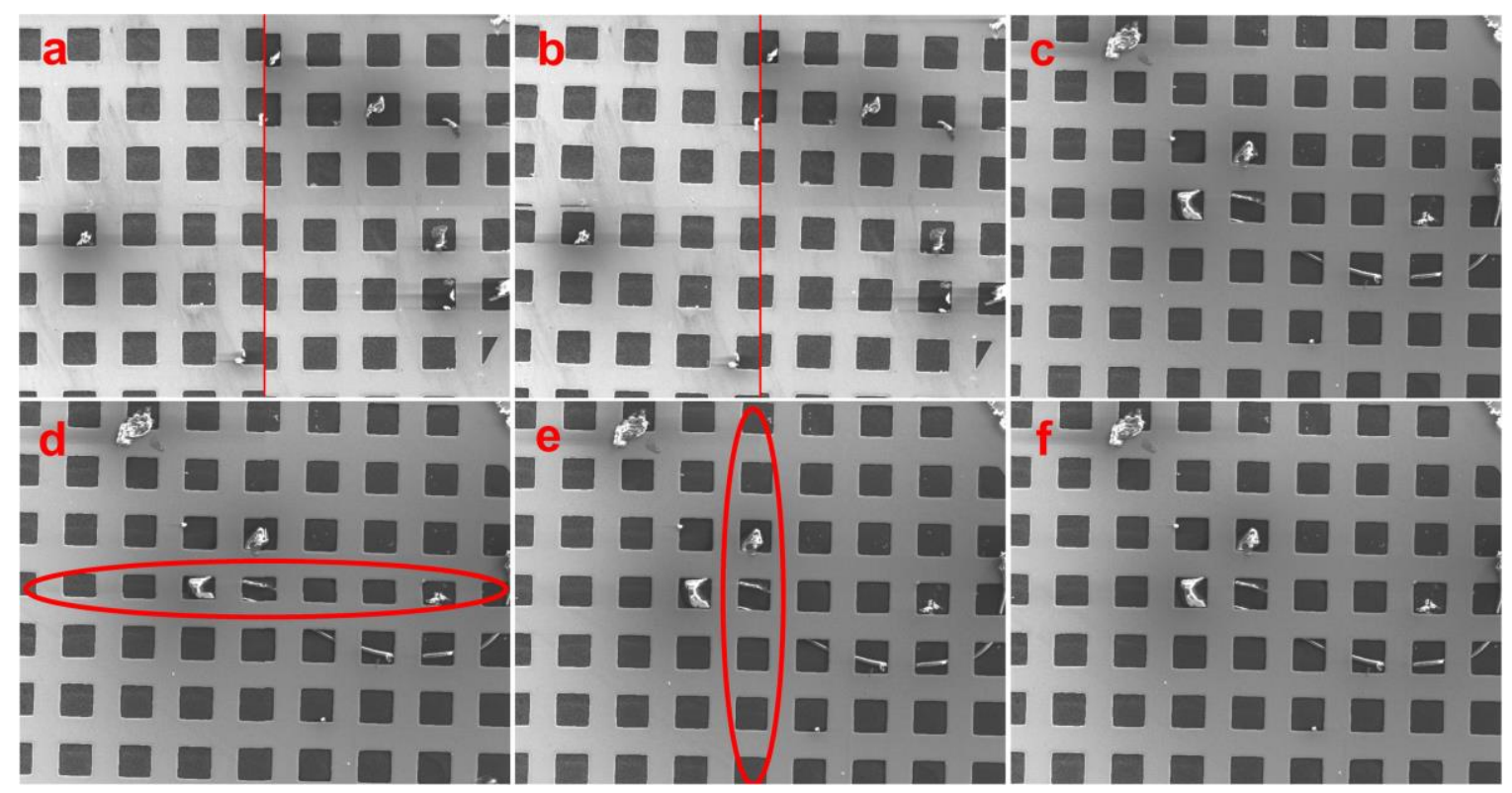

Figure 2. Montage image alignment. The scan rotation angles in a, b, and c are $3^{\circ}, 1^{\circ}$, and $1.6^{\circ}$, respectively. $1.6^{\circ}$ represents a good scan rotation calibration without mismatch in the figure.

\section{References}

[1] PG Kotula, PF Hlava and MR Keenan, Microsc. Microanal. 8 (suppl. 2), 2002, p. 1554CD 